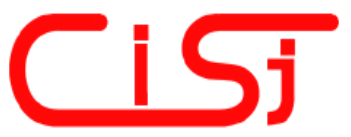

\title{
SMART SILICON SENSOR - MAGNETIC COMPASS ON THE BASE OF A 3-D HALL STRUCTURE
}

\author{
Konstantin Dimitrov \\ Institute of Control and System Research of the Bulgarian Academy of Sciences \\ PO Box 79, BG-1113 Sofia, Bulgaria; e-mail: kdimitrov@icsr.bas.bg \\ www.icsr.bas.bg
}

\begin{abstract}
In this paper is discussed some of the trends in the industrial designs of smart sensor systems. High performance sensor systems have been implemented by combining the sensing and signal processing functions on the same chip. A very promising magnetic compass for Earth magnetic field measurement is created on the base of a 3-D Hall structure.
\end{abstract}

Keywords: Smart sensors, Hall sensors, magnetic compass.

\section{INTRODUCTION}

This paper discusses some state-of-the-art examples of high performance industrial sensor systems and especially magnetic compass realization as smart magnetic sensor. The low transducer cost in combination with the continuous generation of the new ideas has led to a steady growth of the intelligence on a single integrated circuit (IC). Complete sensor systems have been realized on a single chip where traditional functions, such as signal conditioning, analog to digital conversion (A/D), data communication and the generation of reference and compensating signals are no longer performed by separate components, but are all integrated on the same IC. This reduces overall system costs while improving the sensor performance at the same time. Costs can even be further reduced if the IC technology allows for integration of the sensor device in the same substrate. Almost certainly, the best example is the smart temperature sensors [1-3]. Inaccuracy can be as small as $\pm 2{ }^{\circ} \mathrm{C}$ over the temperature range of -60 ${ }^{\circ} \mathrm{C}$ to $+150{ }^{\circ} \mathrm{C}$. Single chip smart temperature sensors are now available as less as possible price (depending mainly from the technologies) in which the temperature sensor, the interfacing electronics, the $\mathrm{A} / \mathrm{D}$ conversion, calibration circuits and bus interface are all ingeniously embedded in the design. Similar technologies are already available or emerging of which, among others, acceleration, capacitive, flow and magnetic field sensors have very high potential.

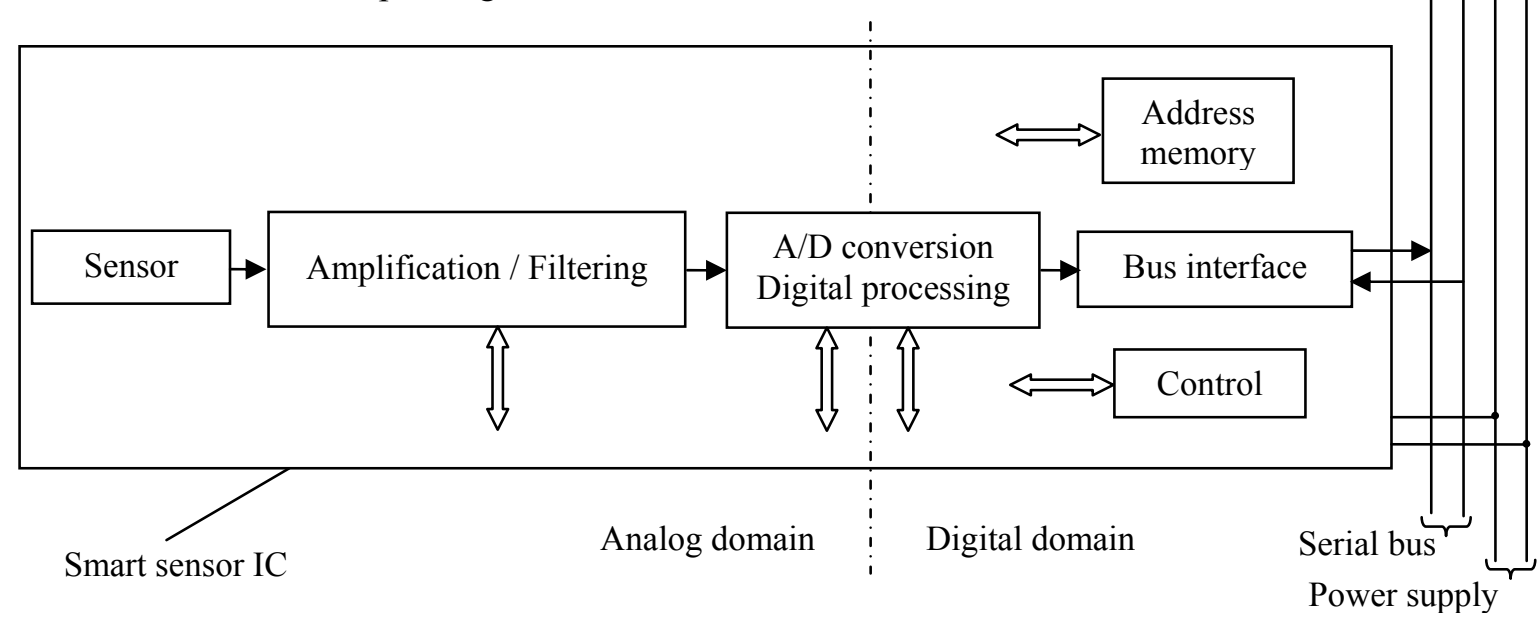

Fig. 1 Basic functional blocks in a smart sensor IC. 
Figure 1 shows a general hardware configuration of such a smart sensor system [4-6]. Smart sensor as all is a device which is added a simple interface that allows connecting the sensor to the serial bus without causing any incompatibility or difficulty to adapt the new element to the bus. Although it is possible to combine a smart sensor with a microcontroller on the chip, it is rarely done because of unnecessary system complexity, high current consumption, interference, incompatible IC process requirements, etc.

The aim of the paper is to introduce hardware configuration and topology of the smart systems as all and especially smart magnetic systems. The focus is put on the sensing element - 3-D Hall sensor important part of the system. In this way, Chapters 1-3 give overview of smart systems and sensor buses.

\section{INTERFACING MICRODEVICES}

In view of their remarkable characteristics, it can be seen that such smart sensors will be used extensively wide spread number of applications if an adequate solution is found to reduce the design cost and simplify the electrical interfacing.

The problem with the interfacing smart sensors, as requisite to manufacture and integrate fully functional microsystems, can be separated in the following two levels: the electronic interface and the technological interface that permits to interconnect different types of microsensors and their associate circuitry.

For the electronic interface, two main interconnection architectures exist: a classical architecture, where the sensed data are multiplexed, conditioned and digitized using a dedicated microcontroller, and a distributed architecture where a bus organized system is used. The second approach introduces the advantages of modularity and interchangeability as it permits a flexible interconnection applicable to different sets of microsystems. On Figure 2 is presented distributed architecture for the electronic interface [7].

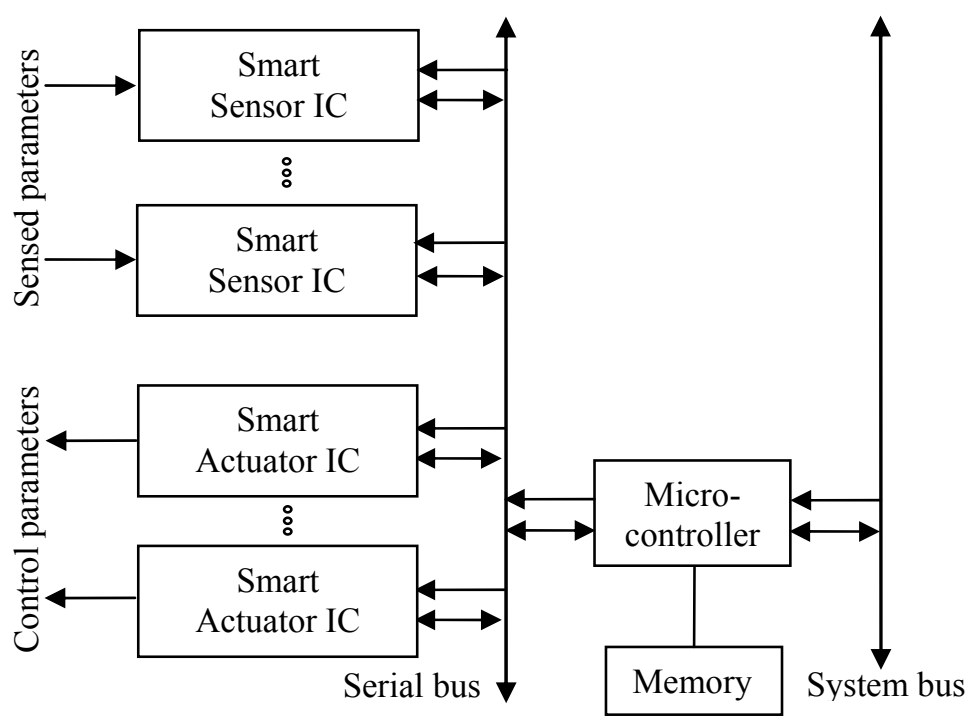

Fig. 2 Distributed architecture for the electronic interface.

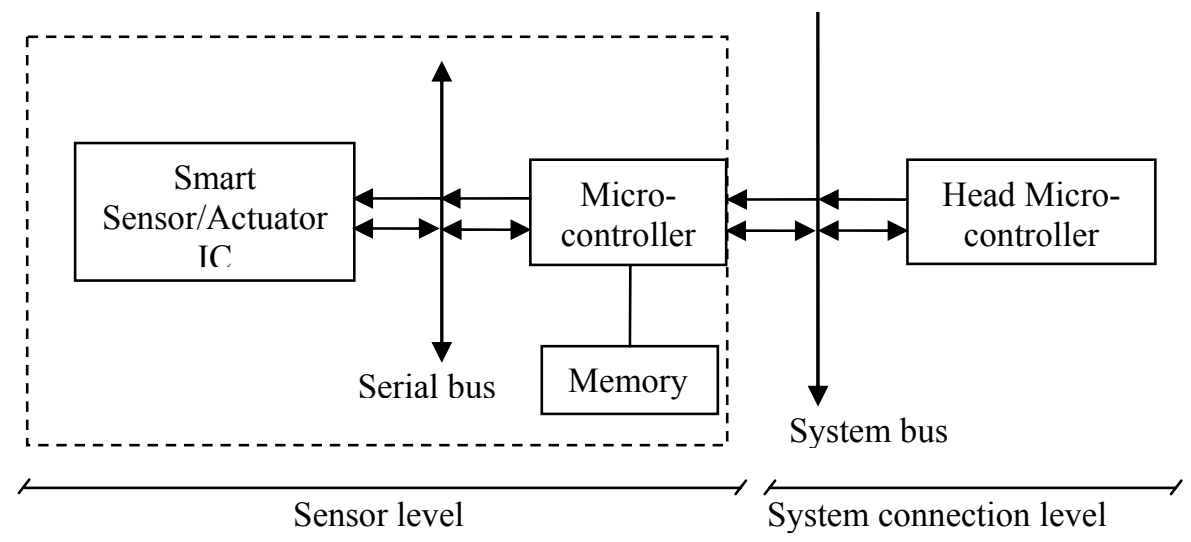

Fig. 3 Topology of the communication system. The means of arrows are as follow:

$$
\leftarrow \text { - SCL (serial clock line) and } \leftrightarrow \text { SDL (serial data line); }
$$




\section{BUS SENSOR DEVELOPMENT}

The serial bus has to be a bus that allows transmitting commands, addresses and data. It has to permit the intercommunication between sensors, actuators and a dedicated microcontroller, in such a way that they can communicate to react to the input events received - sensor level connection, Figure 3. The terminals of the communication system will be sensors and actuators and the device that make the join, the microcontroller. There is an enormous variety of low-cost microcontrollers available. Therefore, for smart sensors it is sufficient to embed the simplest form of control, processing and memory while being able to communicate with any microcontroller through a standard serial bus. In the microcontroller there is to be a control algorithm to give the appropriated commands to sensors and allow them the response in real time and also it would permit to add local processing with the data obtained by the sensors before to be transmitted to the main host - system connection level, Figure 3.

To make a real time system, the implemented drivers have to give a quick response, so the ideal communication will be at least $1 \mathrm{MHz}$ [7]. This low rate of transmission is due to the low volume of data if is compared the application with another one that requires a high volume of data. With this serial bus it will be suitable to be integrated discrete sensors that will measure parameters with a low variation rate.

For the designers is very important task to be chosen communication bus protocol and the appropriated interface for all the elements have been interconnected with the serial bus. These elements are smart sensors (slaves) and the microcontroller (master), to prove the reaction of the final communication elements. These drivers will allow giving certain intelligence to the sensor, making smart sensor [7-9].

In the one chip solution low-cost bus formats, such as Inter IC bus (I2C), the Integrated smart sensor bus (IS2), the Michigan serial Standard, MICROWIRE, SPI have been developed for use in smart sensor systems. These standards have been successful applied in many domestic and industrial applications. The IS2 is a new, simplified sensor bus, which allows both digital and analogue signal communication. A single interface chip is able to switch analogue voltage/current, frequency/duty cycle or bit streams and digital codes on the bus wires. This has made possible to design a very lowcost and easy to build bus system. With respect to these sensor buses it seems that the SPI, MICROWIRE and I2C protocols to have become the industry standards $[4,7]$.

\section{MAGNETIC FILED SENSORS}

Magnetic sensors are used for the measurement of magnetic fields. These fields are caused by various sources, such as a permanent magnet, the earth, a current or a biological system. A wellknown application of magnetic sensors is the indirect measurement of mechanical parameters using a small moving permanent magnet in combination with the sensor.

Early silicon Hall plates measured the magnetic field in only one direction. Nowadays, research on silicon magnetic sensors is concentrated on the three-dimensional measurement of magnetic fields. Experimental devices that measure all three components of the magnetic field are currently being realized with Hall plates and magnetotransistors.

Despite the capabilities of (digital) signal processing, smart sensor accuracy primarily depends on the accuracy of sensing element. Therefore, a main part of the design effort goes into the selection of the physical effect and into the design of the sensor structure. Magnetic fields can be measured with various types of sensors using, among others, the Hall effect, the magnetoresistive effect and the fluxgate effect. Despite the higher sensitivity of the sensors exploit others effects, magnetic sensors based on the Hall effect are preferable. The main reason is that this is a well defined and predicable phenomenon with clear galvanomagnetic behavior. The demand for low-cost high performance solutions force the designs towards using the Hall effect, because it is the only effect that can be implemented in a standard CMOS technology.

\subsection{Hall Effect Sensors}

Early Hall plates were fabricated using a rectangular piece of semiconductor material $(\mathrm{Si}, \mathrm{Ge}$, $\mathrm{AlSb}, \mathrm{InSb}$, etc.). Later, Hall plates were integrated in silicon IC processes. Since then, integrated Hall plates have been proved continuously [10].

To create a single chip that can measure the three dimensional magnetic field vector using Hall plates, it is necessary to extend the classical Hall plate. One possibility is to use one planar Hall plates with two vertical Hall plates. Because vertical Hall plates are less sensitive that planar Hall plates, the sensitivity for the different components of the magnetic field vector are not equal.

Typical sensitivities of Hall plates fabricated in standard CMOS technology are in the range of 100 $200 \mathrm{mV} / \mathrm{VT}$. Most precise applications require magnetic field strengths to be measured in the signal range of $50 \mu \mathrm{T}$ (Earth magnetic filed- minimum detected induction $B_{\min }$ ) up to $250 \mathrm{mT}$. The left border of this range makes very difficult the use of CMOS Hall effect sensors. Most commercially 
available Hall sensors typically operate over ranges that start at $1 \mathrm{mT}$. The main problem for realizing a low-cost compass using silicon Hall sensors is its offset and, even more important, the drift of the sensor offset.

\subsection{Offset and Drift of Hall Sensors}

The perfect Hall sensor does not produce an output signal (voltage/current) when a magnetic field is absent. In practice all Hall sensors have offset as a result of material inhomogenities, alignment error of the sense contacts and because of the mechanical construction of the sensor. This offset can be compensated for be means of calibration as long as the offset is constant. Unfortunately, there are a lot of effects that instigate the offset. Mechanical stress, temperature variations and aging cause the sensor offset to fluctuate. This requires frequent re-calibration of the sensors, which is unwanted. Furthermore, realistic signal levels of a few $\mu \mathrm{V} / \mu \mathrm{A}$ become completely masked by offset levels that can be than tens of $\mathrm{mV} / \mathrm{mA}$. This undesired effect can be ignored with some offset compensation technique. A lot of papers devoted on this problem have launched so-called spinningcurrent method for reducing the offset. This will be the best choice of designers if there are used the simplest Hall elements. In this research work a single Hall plate for 3-D detection of magnetic field is used- spinning-current method is not applicable.

In this case analog front-end integrated circuit for the automatic offset compensation technique is preferred [11]. When a good CMOS analog design and layout practice is followed and no calibration technique is used, offsets are generally in the range of $\pm 5 \mathrm{mV}$. Several automatic calibration techniques are proposed to reduce the amount of this input offset [12-14]. Usually, the auto-zero methods need passive components like a capacitor with analog switches or require a specific architecture to achieve an offset compensation. The implementation does not require any special trimming process and no offchip components. The compensation uses an additional small area 6 bits current mode digital to analog convertor, a comparator and a digital block achieving a successive approximation algorithm. Automatic offset compensation technique is capable of reducing the offset down to $10 \mu \mathrm{T}[11,15]$.

\section{ELECTRONIC COMPASS}

\subsection{A 3-D Hall Sensor}

The integration of more than one measuring function in the active region of the silicon sensors is a recent tendency in the measurement of the individual orthogonal components of the magnetic field vector $\boldsymbol{B}$.

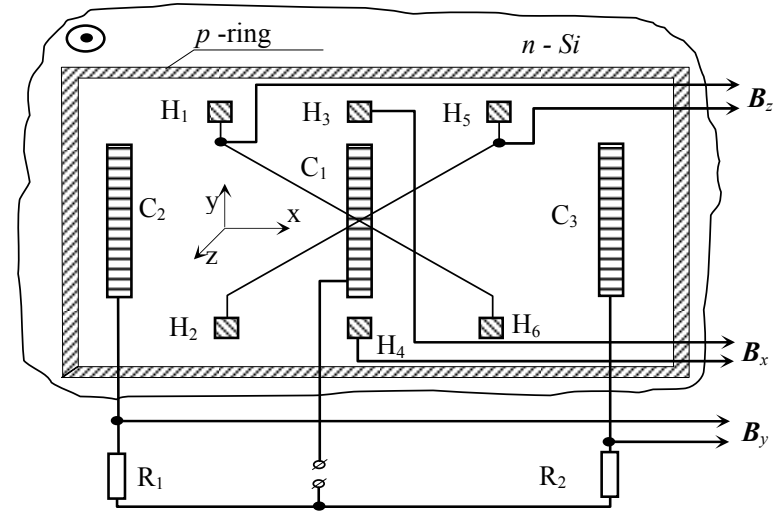

Fig. 4 3-D Hall structure in the base of the smart compass

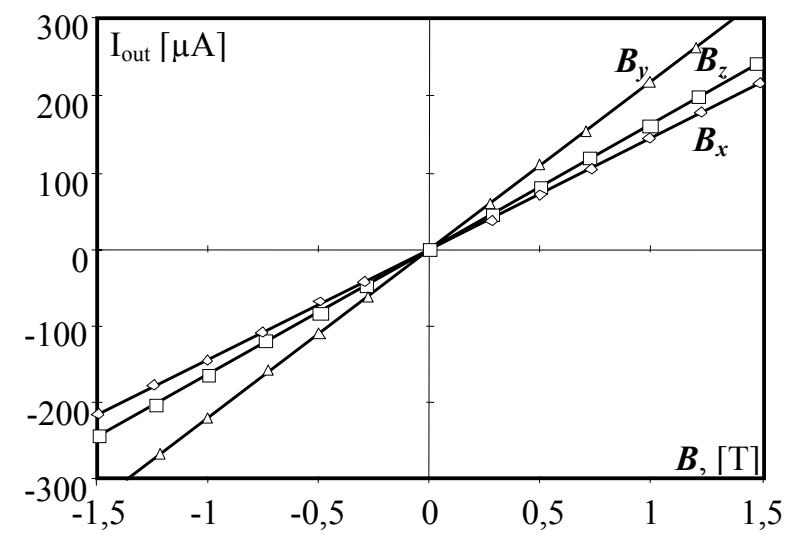

Fig. 5 Output characteristics for $B_{x}, B_{y}$ and $B_{z}$ channels for the 3-D Hall microdevice at supply current $I_{C I}=10 \mathrm{~mA}$.

This approach is used for design of 3-D magnetometers on the base of Hall effect principle, Figure 4. There advantages are: simplified device which maximally approaches the original physical cause (this cause is responsible for the measurement of the Hall effect respective vector component) and simultaneous on line 3-D measurement by separate differential outputs. Thus, the cross-sensitivity of the magnetometer is substantially reduced. The electronic version of a classical compass based of a 3-D silicon Hall structure is created, Figure 4 [15]. Design of the chip, technology used, and the manufacturing process are not a subject of this paper. There are presented some of the important characteristics of the 3-D Hall vector sensor.

Figure 5 shows the output characteristics $I_{\text {out }}(\boldsymbol{B})$ of the 3-D magnetic sensor without any electronic amplification, where the inevitable offset of the channels is nullified in advance by the used circuitry.

It has been established experimentally that, despite the simplified device design of the sensor, the cross-sensitivities are about three orders of 
magnitude lower than the respective channel sensitivities. The output currents $\Delta I_{\text {out }}\left(\boldsymbol{B}_{x}\right), \Delta I_{\text {out }}\left(\boldsymbol{B}_{y}\right)$ and $\Delta I_{\text {out }}\left(\boldsymbol{B}_{z}\right)$ exhibit a linear and an odd dependence on the magnetic field, their non-linearity factor (NL) in the range $-100 \mathrm{mT} \leq \boldsymbol{B} \leq 100 \mathrm{mT}$ being about $0.4 \%$ and in the interval $-1 \mathrm{~T} \leq \boldsymbol{B} \leq 1 \mathrm{~T}$ the NL does not exceed $1 \%$.

A measuring setup has been chosen to contain operational amplifiers, keeping equal voltage conditions on the top of the chip to minimize the lateral parasitic surface currents. These are invoked by the differential potential levels of the three Hall voltages generated simultaneously by the $\boldsymbol{B}_{\boldsymbol{x}}, \boldsymbol{B}_{\boldsymbol{y}}$ and $\boldsymbol{B}_{z}$ components. If they are not kept at equal voltages, false signal may occur. This is why the three differential outputs are Hall currents.

Figure 6 and 7 show the angle dependencies of Hall currents at rotation of the microsensor around the $x$ axis and $y$-axis respectively.

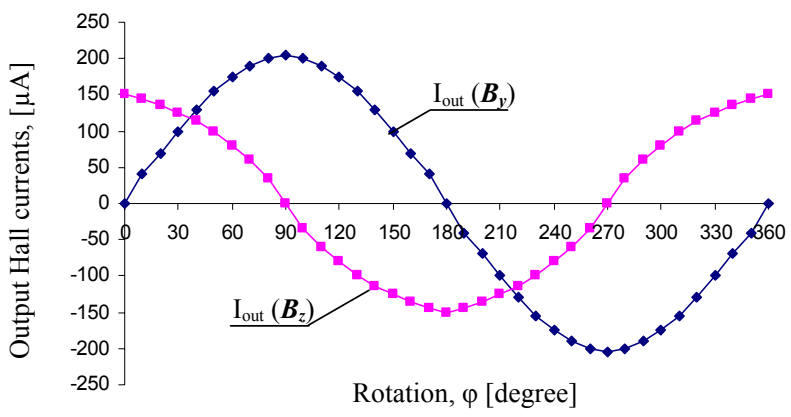

Fig. 6 Angle dependencies of the output Hall currents at rotation of the sensor around the $x$ - axis. The homogenous magnetic field $B_{z}$ is fixed along the $z$ - axis with a value of $B_{z}=1 \mathrm{~T}$; supply current is $I_{\mathrm{C} 1}=10 \mathrm{~mA}$.

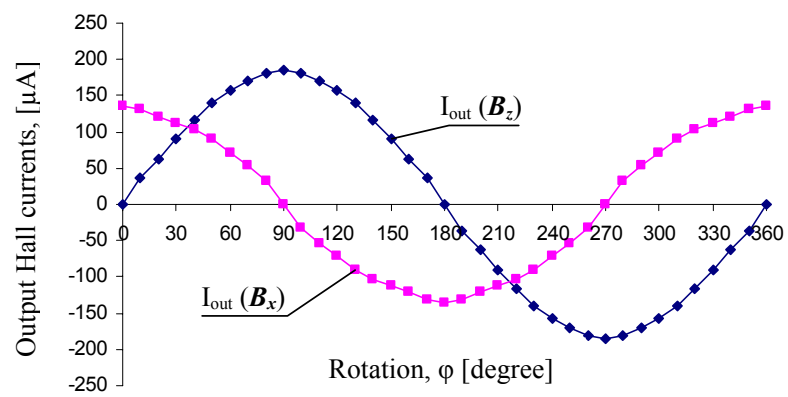

Fig. 7 Angle dependencies of the output Hall currents at rotation of the sensor around the $y$-axis. The homogenous magnetic field $B_{x}$ is fixed along the $x$-axis with a value of $B_{x}=1 \mathrm{~T}$; the supply current is $I_{C 1}=10 \mathrm{~mA}$.

The magnetosensitivities of the three channels at $I_{C l}=10 \mathrm{~mA}$ reach $130 \mu \mathrm{A} / \mathrm{T}$ for $\boldsymbol{B}_{x}, 220 \mu \mathrm{A} / \mathrm{T}$ for $\boldsymbol{B}_{\boldsymbol{y}}$ and $150 \mu \mathrm{A} / \mathrm{T}$ for $\boldsymbol{B}_{z}$, respectively.

As expected the output currents were shifted in phase by 90 degrees. The output signal follows very well the relations $\boldsymbol{I}_{\text {out }} \sim \boldsymbol{B} \cdot \cos \varphi$ and $\boldsymbol{I}_{\text {out }} \sim \boldsymbol{B} \cdot \sin \varphi$.
The temperature coefficient of the magnetosensitivities for all three channels is $0.1 \% /{ }^{\circ} \mathrm{C}$. The absolute value of the magnetic vector $\boldsymbol{B}$ is given by the well-known operation: $|B|=\sqrt{B_{x}^{2}+B_{y}^{2}+B_{z}^{2}}$. The active volume of the 3-D Hall microsensor is about $250 \mu \mathrm{m} \times 220 \mu \mathrm{m} \times 100 \mu \mathrm{m}$. The power consumption at $T=300 \mathrm{~K}$ is in the range $W \leq 30$ $\mathrm{mW}$ and does not disturb the accuracy of the device.

\subsection{Smart Compass}

The magnetic field of the Earth is about $50 \mu \mathrm{T}$, which requires an additional amplification $[15,16]$. In previous realization of an electronic compass were used two pairs of ferrite cone flux concentrators of the magnetic field for each of the two $x$ and $y$ measuring channels, which amplify the outputs about 45 times [15]. The flux concentrators were situated over the ceramic insulating carrier. Besides, the whole system is placed in a medium of liquid nitrogen under temperature $T=77 \mathrm{~K}$, which additionally enhances the output signals 4 times.

The output signals are amplified by low noise operational amplifiers. As a result, the experiments that have been carried out in open-air and far from metal objects, have shown resolution of the geomagnetic field about $5 \mu \mathrm{T}$ and accuracy of about $3 \%$ at supply current $\boldsymbol{I}_{C l}=20 \mathrm{~mA}$. Smart compass realization has shown resolution of the geomagnetic field of about $1 \mu \mathrm{T}$ and accuracy better than $1 \%$, respectively.

In case the compass is mounted in the neighborhood of metal objects it is recommended to compensation the system for the distortion of the Earth magnetic field. The calibration procedure consists of a calibration reading of a full circle made in the earth magnetic field and fitting the measured cosine and sine signals to ideal polar coordinates. The fir parameters are then stored and used in headings calculations.

The 3-D Hall magnetometer overcomes many of the drawbacks associated to magneto-resistive and magneto-inductive sensor technologies. The smart sensor system does not require calibration and recalibration, it does not need demagnetizing coils, and it is insensitive to magnetic field overloading. Because of the capability of standard CMOS technology, the smart magnetic compass system is equipped with an on-chip instrumentation amplifier, $\mathrm{A} / \mathrm{D}$ converter and logic to provide a convenient digital output signal.

Various static compensation techniques have been applied to the Hall sensor to result with extremely low offset levels. This sensor compensates for mechanical (metal/packaging) stresses, doping gradients and mask misalignments 
caused by processing variations. The sensor also compensate for thermally induced signals. Furthermore, the sensor compensates for magnetic induced signals that are caused by the current-drive lines that are connected to the sensor element. In addition, various dynamic offset cancellation techniques have been applied to the sensor and instrumentation design to result with offsets that are well below $10 \mu \mathrm{T}$. The bitstream is processed by onchip logic and is made available to the user through pulsewidth module digital output, RS 232 or SPI/MICROWIRE.

\section{CONCLUSIONS}

This smart sensor IC is a development towards a smart compass on the base of 3-D Hall structure. It contains some basic signal processing to amplify the low-level sensor signal to a level where it can be handled by an A/D converter. The output of the smart compass can be connected to the some serial interface of the microprocessor.

The smart sensor system can be also successful applied as a navigation element controlling the course of moving apparatuses like missiles, submarines, satellites, aircrafts, etc. In this case, the preliminary given moving directions is fixed through the measurement circuit as a zero output signal in the smart compass.

\section{REFERENCES}

[1] Smartec, Datasheet SMT160-30, An integrated temperature sensor with duty-cycle output.

[2] Analog Devices, Datasheet AD 7416, 10-bit digital temperature sensor with I2C, SPI and MICROWIRE bus interface.

[3] Philips, Datasheet LM 75 A, 11-bit digital temperature sensor with I2C bus interface.

[4] P. de Jong, F. Riedijk and J. van der Meer, Smart Silicon Sensors- Examples of Hall effect Sensors, IEEE Sensors 2002, June 11-14, 2002, Orlando, Florida, USA.

[5] G.C.M. Meijer, Concepts and focus point for intelligent sensor systems, Sensor and Actuators, A, Vol. 41-42 (1994) pp. 183-191.

[6] J. H. Huising, F. R. Riedijk, G. van der Horn, Developments in integrated smart sensors, Sensors and Actuators, A, Vol. 43 (1994) pp. 276-288.

[7] C. Ferrer, B. Lorente, Smart sensors development based on a distributed bus for microsystem applications, Proc. of the SPIE's First Intern. Symp. On Microtechnologies for the New Millenium 2003: Smart sensors, actuators and MEMS. Maspalomas, Gran Canaria, Spain, 19-21 May 2003.
[8] K. Dimitrov, Magnetic sensor systems, Proc. of the $14^{\text {th }}$ Scientific Symposium Metrology and Metrology Assurance 2004, September 14-18, 2004, Sozopol, Bulgaria, pp. 180-183.

[9] K. Dimitrov, Smart magnetic sensors, Annual Proc. of the TU - Varna 2004, October 7-9, 2004, Varna, Bulgaria, pp. 134-138.

[10] R. Popovic, Hall Effect devices: Second Edition, IOP 2004.

[11] M. Kayal, E. Chevallaz, F. Burger R. Popovic, Magnetic Angular encoder using an automatic offset cancellation technique, IEEE Sensors 2002, June 11-14, 2002, Orlando, Florida, USA, pp. 767-770.

[12] Ch. Enz and G. Temes, Circuit techniques for reducing the effects of Op-Amp Imperfections: Autozeroing, correlated double sampling, and chopper stabilization, Invited paper, IEEE $J$. Solid-State Circuits, Vol. 84, No 11. November 1996.

[13] M. Kayal, R. T. L. Saez and M. Declercq, An Automatic Offset Compensation Technique Applicable to Existing Operational Amplifier Core Cell, Proc. of IEEE Custom Integrated Circuits Conference, 1998, pp. 419-422.

[14] Z. Randjelovic, M. Kayal, R. S. Popovic and H. Blanchard, Highly sensitive Hall magnetic sensor microsystem in CMOS technology, Journal of Solid-State Circuits, Vol. 37, No 2, February 2002.

[15] Ch. Roumenin, K. Dimitrov, A. Ivanov, Integrated vector sensor and magnetic compass using a novel 3D Hall structure, Sensors and Actuators, A, Vol. 92 (2001) pp. 119-122.

[16] Xensor Integration, Datasheet EMF-03, Earth magnetic field sensor.

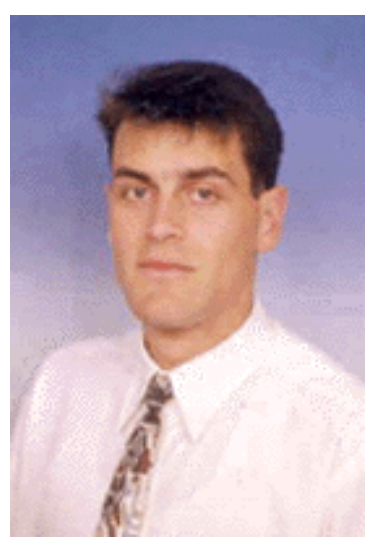

Konstantin V. Dimitrov, is received his $\mathrm{Ph}$. D. degree in measurement science from the Institute of Control and System Research (ICSR) with the Bulgarian Academy of Sciences (BAS), Sofia, Bulgaria (2002). Since July, 2002 he is Research Fellow at Sensor Systems and Modeling Department at ICSR - BAS. His research interests are focused on modeling and simulation of Hall effect devices, Smart sensors and Microsystems for magnetic field and temperature. 